\title{
Relationship Between the Chondrocyte Maturation Cycle and the Endochondral Ossification in the Diaphyseal and Epiphyseal Ossification Centers
}

\author{
Ugo E. Pazzaglia, ${ }^{1 *}$ Terenzio Congiu, ${ }^{2}$ Valeria Sibilia, ${ }^{3}$ Francesca Pagani, ${ }^{3}$ \\ Anna Benetti, ${ }^{4}$ and Guido Zarattini ${ }^{1}$ \\ ${ }^{1}$ Department of Medical and Surgical Specialties, Radiological Sciences and Public Health, \\ University of Brescia, Brescia, Italy \\ ${ }^{2}$ Department of Surgical and Morphological Sciences, University of Insubria, Varese, Italy \\ ${ }^{3}$ Department of Medical Biotechnology and Translational Medicine, University of Milan, Milan, Italy \\ ${ }^{4}$ Department of Clinical and Experimental Sciences, University of Brescia, Brescia, Italy
}

\begin{abstract}
The chondrocyte maturation cycle and endochondral ossification were studied in human, fetal cartilage Anlagen and in postnatal meta-epiphyses. The relationship between the lacunar area, the interterritorial fibril network variations, and calcium phosphorus nucleation in primary and secondary ossification centers were assessed using light microscopy and scanning electron microscopy (SEM) morphometry. The Anlage topographic, zonal classification was derived from the anatomical nomenclature of the completely developed long bone (diaphysis, metaphyses and epiphyses). A significant increase in the chondrocyte lacunar area was documented in the Anlage of epiphyseal zones 4 and 3 to zone 2 (metaphysis) and zone 1 (diaphysis), with the highest variation from zone 2 to zone 1. An inverse reduction in the intercellular matrix area and matrix interfibrillar empty space was also documented. These findings are consistent with the osmotic passage of free cartilage water from the interfibrillar space into the swelling chondrocytes, which increased the ion concentrations to a critical threshold for mineral precipitation in the matrix. The mineralized cartilage served as a scaffold for osteoblast apposition both in primary and secondary ossification centers and in the metaphyseal growth plate cartilage, though at different periods of bone Anlage development and with distinct patterns for each zone. All developmental processes shared a common initial pathway but progressed at different rates, modes and organization in diaphysis, metaphysis and epiphysis. In the ossification phase the developing vascular supply appeared to play a key role in determining the cortical or trabecular structure of the long bones. J. Morphol. 000:000-000, 2016. () 2016 Wiley Periodicals, Inc.
\end{abstract}

KEY WORDS: bone development; cartilage Anlage; chondrocyte maturation cycle; mineral deposition; endochondral ossification

\section{INTRODUCTION}

During human embryonic development, the limb buds appear at approximately 4 weeks of age (3.5 $\mathrm{mm}$ in length, Streeter stage 12). The upper limb buds can be observed slightly earlier than the lower ones. Between the 6th and 9th weeks of development, the cartilage Anlagen of the major bones are formed (Streeter, 1951; Uhthoff, 1990). The initial ossification in the late embryonic period (O'Rahilly and Gardner, 1972) continues throughout the fetal and early postnatal periods. The secondary ossification centers of the epiphyses appear after birth in humans (Vogt and Vickers, 1938; Noback, 1944; Greunlich and Pyle, 1959; Caffey, 1973). The cartilage growth model is characterized by the chondrocyte maturation cycle, which, combined with matrix synthesis, represents the first step in endochondral ossification. These processes are differently distributed in the Anlage zones and lead to the formation of the diaphyseal cortex or to the trabecular bone of epiphyses and metaphyses (Ballock and O'Keefe, 2003).

The most frequently used model in the study of the chondrocyte maturation cycle and endochondral ossification is the metaphyseal growth plate in experimental animals (Wilsman et al., 1966a,b; Hunziker et al., 1987; Farnum and Wilsman, 1993), where there is an evident ordered and sequential progression of the two aspects, that control the longitudinal growth of the bone. The

\footnotetext{
Conflict of interest: None of the authors had conflicts of interests.

Contract grant sponsor: "Medical and Surgical Specialties, Radiological Sciences and Public Health Department” of the University of Brescia.

*Correspondence to: Ugo E. Pazzaglia, Department of Medical and Surgical Specialties, Radiological Sciences and Public Health, University of Brescia, Brescia, Italy.

E-mail:ugo.pazzaglia@asst-spedalicivili.it
}

Received 19 November 2015; Revised 16 May 2016; Accepted 26 May 2016.

Published online 00 Month 2016 in Wiley Online Library (wileyonlinelibrary.com). DOI 10.1002/jmor.20568 


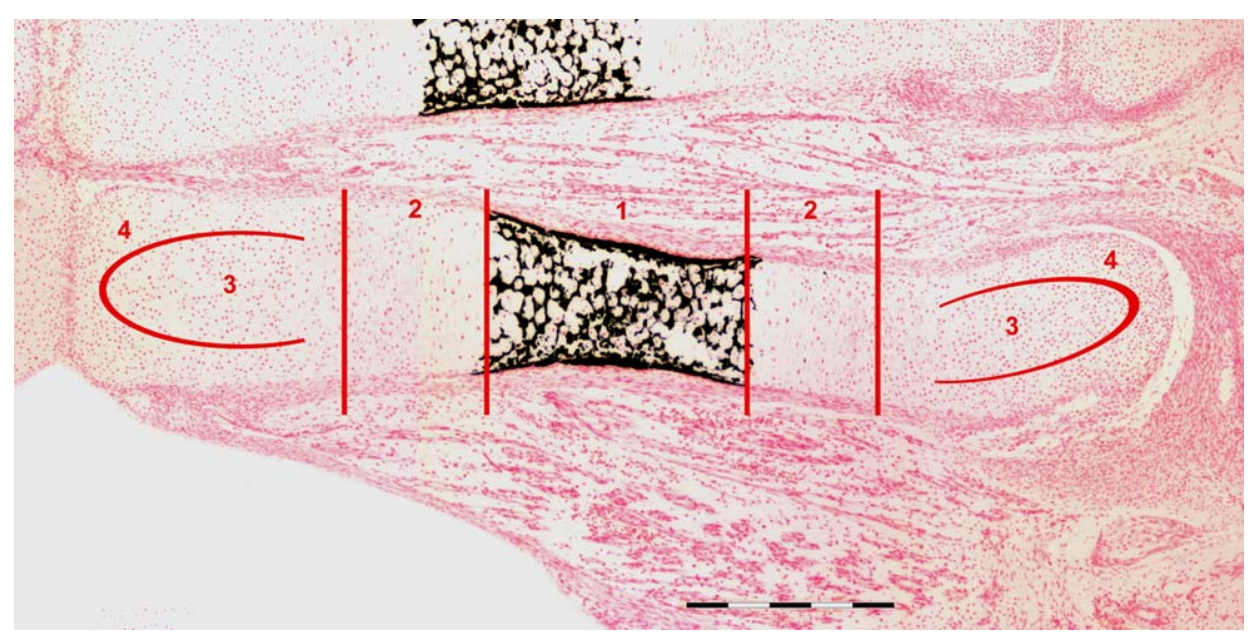

Fig. 1. Human fetal, 2nd metacarpal, 20th week (Von Kossa-neutral red/bar $=500 \mu \mathrm{m})$. Skeme showing the boundaries used for zones definition and morphometry. 1-mid-shaft diaphysis, 2proximal and distal diaphysis, 3-central epiphysis; 4-peripheral epiphysis; see text for details.

fetal cartilage Anlagen exhibit an earlier developmental stage than the metaphyseal growth plate does. The proliferation pattern of the cells is not ordered in this way but is also oriented in columns in zone 2, which corresponds to the metaphysis of the later bone development. The threedimensional symmetric or asymmetric distribution of growth only defines the rough globular shape of a short bone or the elongated shape of a long bone (Pazzaglia et al., 2011).

This study assesses the chondrocyte lacunar area rather than volumetric parameters of the cell to improve measurement accuracy because lacunae exhibit fewer processing artifacts than chondrocytes do. The number of lacunae, their density, the total lacunar area and the intercellular matrix area can be compared in histological fields of equal size in the cartilage Anlage zones. Mineral deposition on the cartilage matrix and the related endochondral ossification is correlated with the chondrocyte maturation cycle (Bonucci, 2007). The latter begins at different time points in different ossification centers of the same Anlage but with a basic common pattern, that produces a different structural layout in the diaphyseal ossification center, the epiphyseal and the metaphyseal growth plate cartilage. Therefore, the study has been extended to include available histological slides of early postnatal human bones, in which the epiphyseal and metaphyseal chondrocyte maturation cycle can be evaluated in corresponding zones in the two age groups.

The aims of the study are: (1) to examine the correlation of the chondrocyte maturation cycle (through lacunar size parameters) with the associated interterritorial matrix transformation which leads to calcium phosphorus nucleation; (2) to evaluate chondrocyte morphology definition, distribution and temporal sequence in the Anlage of the ossification centers development; and (3) to compare the structural layouts of diaphyseal and epiphyseal ossification.

\section{MATERIALS AND METHODS Light Microscopy Histology}

The histological material for the study was obtained through a retrospective analysis of the Morbid Anatomy slide archives, where all pregnancy terminations are routinely examined. An initial selection of cardiovascular and brain malformation cases without skeletal dysmorphia (assessed by prenatal ultrasound diagnosis) was made. The pregnancies were terminated between the 16 th and 22 nd week. The examined material did not allow the assessment of fetal length. The inclusion criteria required that slides comprised whole bone cartilage Anlagen or the appropriately oriented proximal/distal area (longitudinal section). Twelve samples (including several Anlagen) were selected. The study protocol was approved by the Council of the Department of Medical and Surgical Specialties, Radiological Sciences and Public Health of the University of Brescia.

All Anlagen of the limbs showed the same cycle of chondrocyte maturation, but its development was delayed in the distal limbs Anlagen compared to the proximal limbs Anlagen. Furthermore, the development of the lower limbs was slightly delayed compared to the upper limbs. Therefore, in fetuses between the 16th and 22nd week the cartilage Anlagen which corresponded to the histological stages $3 \mathrm{~b}-7$ according to the Rivas and Shapiro classification (2002) were examined.

Fifty-nine longitudinal Anlagen of cartilage in histological slides from 12 fetuses satisfied the inclusion criteria: 28 metacarpals and metatarsals; 16 phalanges; 15 humeri, radii/ulnae, femura and tibiae/fibulae. The tissue specimens had been fixed in neutral formaldehyde solution (10\%), dehydrated in a series of increasing ethanol solution concentrations and embedded (undecalcified) in paraffin blocks. Ten micrometer thick sections were produced and stained with hematoxylin-eosine, Alcian blue $(\mathrm{pH} 1)$, PAS and the von Kossa method. Sections were then mounted on glass slides and observed with an Olympus BX51 microscope.

Histology of the meta-epiphyseal postnatal development was obtained from slides of the senior author's educational collection. These include four cases: two proximal tibiae (2 and 3 months after birth) and two proximal humeri (3 and 5 months 


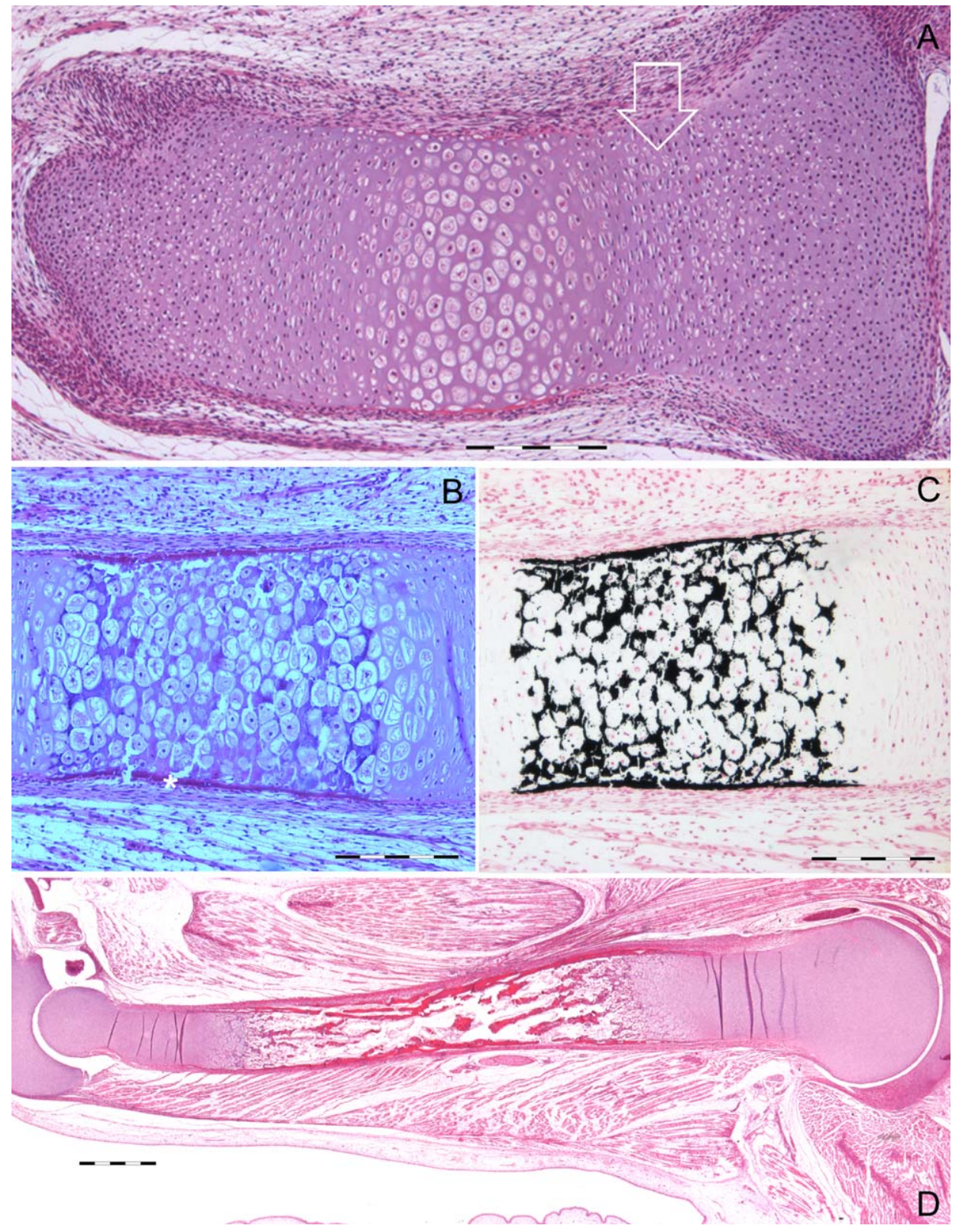

Fig. 2. Human fetal, middle phalanx of 2nd finger. (A) 17th week, hist. stage 3a (hematoxylin-eosin/bar $=200 \mu \mathrm{m}$ ). Chondrocyte hypertrophy in the Anlage zone 1) prior to mineral deposition. The chondrocytes are randomly distributed in both zone 1 and in the epiphyses. In zone 2, cell lacunae appear flattened and aligned longitudinally (arrow). The periosteum surrounds zone 1 and osteoblasts have formed an uncalcified thin layer of the periosteal sleeve. (B) 20th week, hist. stage 4a, 3rd metacarpal (Hematoxylineosin/bar $=200 \mu \mathrm{m})$. The hypertrophic chondrocyte zone 1 is circularly enveloped by a periosteal sleeve of enhanced thickness. A layer of periosteal osteoblasts is present on the external surface. Swollen chondrocytes and mineral deposition are apparent in zone 1. An increased width of zone 2 reveals a more apparent stacking of chondrocyte columns than in A. (C) 20th week, hist. stage 4 a, 3rd metacarpal (Von Kossa-neutral red/bar $=200 \mu \mathrm{m}$ ). The same slide in B documents the mineral deposition on the matrix between the enlarged chondrocyte lacunae. Dark spots inside the lacunae are artifacts. The periosteal sleeve appears densely calcified and does not expand beyond the mineralized zone 1. (D) 22nd week, hist. stage 5a, humerus (hematoxylin-eosin/bar $=1 \mathrm{~mm}$ ). Periosteal apposition and remodeling have formed the diaphyseal cortical bone, whereas the zone 1 mineralized cartilage has undergone resorption. Few primary trabeculae inside the marrow canal are undergoing remodeling. Zone 2 chondrocytes reveal longitudinal columns of the metaphyseal growth plate cartilage. The epiphyses are cartilagineous without inner canals.

after birth). The glass slides had been registered as "normal bone development" by the anatomist who collected the samples, which indicated that the children had died from causes unrelated to bone pathology. Both of the tibiae and one humerus had been decalcified, routinely processed and stained with hematoxylin-eosine. The 5-months-old humerus had been embedded undecalcified in methylmetacrylate and stained with the solochrome method. The postnatal epiphysies area was measured by tracing the epiphyseal contour on a graphic tablet (excluding the growth plate) and counting the number of cartilage canals with a low power stereo microscope (Olympus SZX7, Japan) at $20 \times$ magnification. Individual canals were 


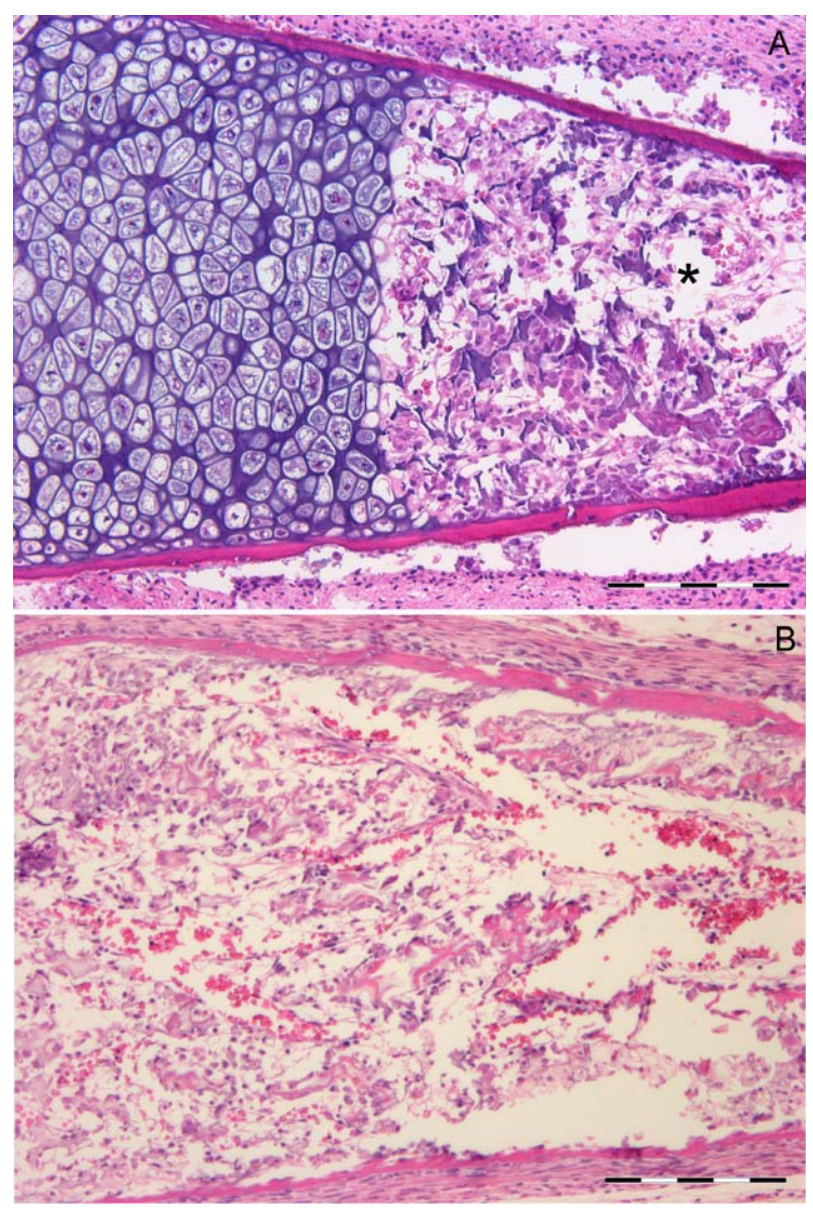

Fig. 3. Human fetal, proximal ulna. (A) 20th week (hematoxylin-eosin/bar $=200 \mu \mathrm{m})$. Mineralized cartilage of zone 1 is undergoing resorption (asterisk) by the vessels from the mid-diaphysis (branches of the central medullary artery). No vessels are observed to pierce through the sleeve from the periosteal network. (B) 20th week, radius (hematoxylin-eosin/bar $=200 \mu \mathrm{m}$ ). Advanced resorption of zone 1 mineralized cartilage and widespread medullary vessel network. There is not calcified cartilage that can serve as a scaffold for secondary trabeculae formation.

considered independently of their shape (circular, elongated or branched).

\section{Light Microscopy Morphometry}

For morphometry a stricter selection of the 59 available cartilage Anlagen (based on orientation) was carried out: 15 whole metacarpals and metatarsals, 10 phalanges, 4 humeri, 2 radii/ ulnae, 2 femura and 2 tibiae/fibulae were selected. The topographic zonal classification used in the current study is derived from the commonly used anatomical nomenclature of the completely developed long bone. This method allows the relationship between the chondrocyte maturation cycle and the ossification process in the human fetal cartilage Anlagen to be evaluated with regard to the lacunar area and the cartilage interfibrillar area. Zones of the whole cartilage Anlage have been demarcated as shown in Figure 1: 1—mid-shaft diaphysis, corresponding to the central part of the cartilage model where the intercellular matrix has been calcified and the outer perimeter delimited by the first periosteal sleeve; 2-proximal and distal diaphysis not yet surrounded by a periosteal bony sleeve, characterized by enlarging chondrocytes and not yet calcified intercellular matrix; 3-central epiphysis; and 4-peripheral epiphysis, corresponding to the outer cartilage layer at approximately $1 / 4$ of the epiphyseal radius.

Morphometry was carried out at $400 \times$ magnification on hematoxylin-eosine stained sections. Randomly selected fields (30 or 20 in each zone) corresponded to an area of $218.63 \times$ $163.63 \mu \mathrm{m}\left(0.035 \mathrm{~mm}^{2}\right)$. The following parameters were assessed: the number of chondrocyte lacunae, the total chondrocyte lacunar area (TCLA), the mean single chondrocyte lacunar area (MSCLA) and the total matrix area (TMA) (calculated from the difference between the total field area and the TCLA). The digital images were obtained using a Color View IIIb camera (Soft Imaging System GmbH, Germany) and processed using Adobe Photoshop, delimiting the outline of each chondrocyte lacuna and staining the area black with the fill function (bucket). The lacunae that were intersected by the field edge were counted and measured. The total number of chondrocyte lacunae, their density, the TCLA, the MSCLA and the TMA were determined with ImageJ (Schneider et al., 2012) using the color threshold function which allows the black stained chondrocyte lacuna to be distinguished from the neighboring matrix.

In the early postnatal specimens (two proximal tibiae and two humeri) 30 randomly selected fields (400× magnification) of the proximal tibial and humeral epiphyses (zone 5) and the corresponding metaphyseal growth plate cartilage hypertrophic cell layer (zone 6) were analyzed using the same parameters as the fetal bone cartilage Anlagen (zone 5 vs zone 3 and zone 6 vs zone 1 ). The early secondary ossification center zone of the older humeral head was excluded from morphometry field sampling and was only used for morphological documentation of the secondary ossification center.

Cartilage canals had not yet formed in any of the examined fetal Anlagen and could only be quantified in the postnatal epiphyses. Canal density was expressed in the total epiphyseal area, which was measured manually using the "Cells" software (Soft Imaging System GmbH, Germany).

\section{Scanning Electron Microscopy}

Unstained histological glass slides from the same paraffin mounts of fetal and early postnatal specimens were processed for observation (Pazzaglia et al., 1987). After deparaffination, slides were dehydrated in increasing concentrations of ethanol solution and subjected to $\mathrm{CO}_{2}$ critical point drying. The slides were then coated with a thin layer of gold in a sputter coater (Emitech) and observed using a Philiphs XL 30 scanning electron microscope. X-ray energy dispersive spectra were obtained from SEM fields $(20.000 \times$ magnification) to document $\mathrm{CaP}$ nucleation.

Assessment of the matrix interfibrillar, empty area was carried out on SEM digital images of the longitudinally sectioned surfaces. These were acquired in the backscattered mode (4.0), with the electron beam perpendicular to the plane of the section, at $20 \mathrm{kV}$ and $10 \mathrm{~mm}$ of working distance. The same magnification of $25.000 \times$ was used for all sections. The cartilage interfibrillar empty space and was measured in 20 randomly selected fields (corresponding to an area of $4.85 \times 3.67$ $\mu \mathrm{m},=17.8 \mu \mathrm{m}^{2}$ ) of metacarpals bone Anlage zone 3,2 , and 1 (with exclusion in the latter of the fields where mineral deposition had already occurred). The total field area, the cartilage interfibrillar empty area and its percentage was assessed with the Image $J$ with the same contrast enhancement in all the fields and using the binary threshold function. The interfibrillar empty area (black) and its percentage of the total field area were compared between zone 3 vs 2 and in zone 2 vs 1 in the same metacarpals. 

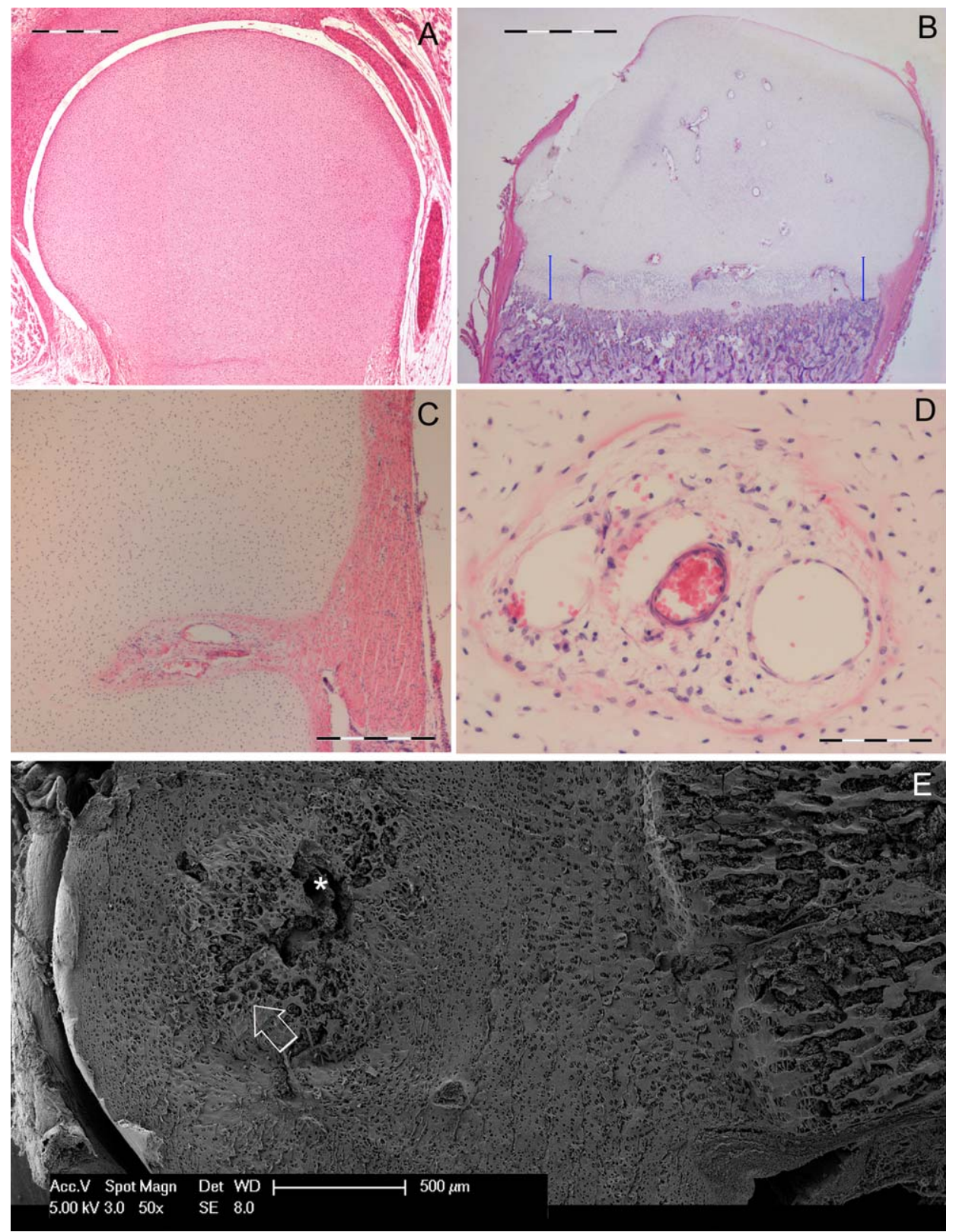

Fig. 4. Human fetal, proximal humerus. (A) 22nd week, (hematoxylin-eosin/bar $=500 \mu \mathrm{m}$ ). Fetal epiphysis prior to the development of cartilage canals. (B) postnatal 3 months old, lproximal humeral epiphysis (hematoxylin-eosin/bar $=4 \mathrm{~mm}$ ). A cartilage canal system has developed in the epiphysis without any evidence of chondrocyte maturation cycle activation. No secondary ossification center is evident. Fetal zone 2 is reduced to the thickness of its own metaphyseal growth plate (defined by the lateral and medial bars). (C) postnatal 3 months old, proximal humeral epiphysis (hematoxylin-eosin/bar $=500 \mu \mathrm{m}$ ). The origin of the cartilage canals system with connective-vascular sprouts entering into the epiphysis. (D) postnatal 3 months old, proximal humeral epiphysis (hematoxylin-eosin/ bar $=100 \mu \mathrm{m}$ ). Vascular lacunae delimited by a single endothelial layer and structured vessels with a "tunica muscularis". Loose connective tissue fills tha canal between vascular lacunae. (E) Postnatal 5 months old proximal humerus (SEM backscattered). The secondary ossification center of the epiphysis reveals hypertrophic chondrocyte columns converging centripetally (arrow). Vessels (asterisk) have reached the center through the epiphyseal cartilage canal system. The metaphyseal cartilage is evident on the right side with the longitudinally and orderly oriented hypertrophic chondrocyte columns. Thin metaphyseal vessels advance between the calcified cartilage septa.

\section{Statistical Analysis}

The accuracy of lacunar outline delimitation (carried out manually) was tested comparing the repeated measurements of total chondrocyte lacunae area (independently obtained by two investigators U.E.P. and T.C.) on a sample of $20 \%$ of the histological fields equally distributed for each zone. The intra-observer and inter-observer variation (Bland and Altman, 2010) has shown an acceptable degree of agreement (95\% confidence interval).

The assessed parameters (TCLA, TMA, chondrocyte lacunae number (CLN), MSCLA) were represented as the mean \pm standard error of the mean (SEM). TCLA and TMA were 
TABLE 1. Postnatal bone epiphyses area, number and density of cartilage canals

\begin{tabular}{lcccccc}
\hline CASE & AGE (months) & EPIPHYSIS & SLIDE & $\begin{array}{c}\text { EPIPHYSIS } \\
\text { AREA }\left(\mathrm{mm}^{2}\right)\end{array}$ & $\begin{array}{c}\text { CART CANALS } \\
\text { NUM }(n)\end{array}$ & $\begin{array}{c}\text { CART CANALS } \\
\text { DENS }\left(\mathrm{n} / \mathrm{mm}^{2}\right)\end{array}$ \\
\hline 1 & 2 & prox and distal tibia & 1.1 & 87.3 & 21 & 0.24 \\
& & & 1.2 & 55.1 & 20 & 0.36 \\
2 & 3 & Proximal tibia & 2.1 & 76.9 & 27 & 0.35 \\
3 & & & 2.2 & 55.6 & 26 & 0.36 \\
4 & 3 & Proximal humerus & 3 & 85.1 & 52 & 0.31 \\
& 5 & Proximal humerus & 4.1 & 144.1 & 33 & 0.27 \\
\hline
\end{tabular}

expressed either as absolute values or as TCLA/TMA. Data were analyzed by one way Analysis of Variance (ANOVA) followed by multiple comparison Bonferroni t-test. Differences between the groups were analysed using an unpaired t-test. The comparison of the matrix interfibrillar empty area (carried out with SEM) among zones 3,2 , and 1 was analyzed with an unpaired Student

t-test. Values of $P<0.05$ were considered statistically significant.

\section{RESULTS}

\section{Light Microscopy Histology}

Between the 16th and 22nd weeks of development, the chondrocyte maturation cycle prior to the commencement of mineral deposition (stage $3 \mathrm{~b}$ ) can be observed in the hands and distal foot phalanges (Fig. 2A), and the next developmental phase (stage 4a) can be observed in the proximal phalanges, metacarpals and metatarsals Anlagen (Fig. 2B,C). Development in the proximal limbs is more advanced than in the distal limb (stage 5a) with a well-structured diaphyseal cortex apparent (Fig. 2D). Therefore, even in the limited fetal time interval of the current study, the differential lag between bone Anlagen development is apparent in a sufficiently ample histological documentation.

The definition of zones 1 and 2 boundaries at stage $4 \mathrm{a}$ is precisely demarcated by the mineral deposition on the cartilage (Fig. 1). The definition of zones 3 and 4 is based on the previously reported topographic criterion.

After mineral deposition on zone 1 cartilage matrix the diaphyseal ossification continues with osteoblasts layer differentiation on the external surface of the Anlage and the development of the periosteal vascular network (Fig. 2B). In all studied Anlagen, the zone 1 calcified cartilage matrix appeared to serve as a scaffold for early osteoblasts apposition but did not take part in further cortex formation. The diaphyseal circumference increases through eccentric, periosteal apposition, while the inner calcified cartilage zone 1 undergoes chondroclast resorption and substitution by the developing marrow vascular network and by red marrow tissue (Fig. 3A,B). The periosteum advances both proximally and distally towards zone 2, where chondrocytes are becoming hypertrophic and align longitudinally. In the proximal limb Anlagen (humerii, radii/ulnae, femura, tibiae, and fibulae), a medullary vascular network has been formed by the branches of the central nutritive arteries. The hypertrophic chondrocyte and the calcified matrix of zone 1 are undergoing resorption.

Cartilage canals have not been observed in fetal Anlage of the meta-epiphyses at up to 22 weeks of age (Fig. 4A), but they are commonly observed in the early postnatal period before the appearance of the secondary ossification centers (Fig. 4B and Table 1). Cartilage canals enter into the cartilagineous epiphyses from the peripheral border (Fig. 4C) or across the metaphyseal growth plate. They can only contain loose connective tissue or vascular lacunae surrounded by a simple endothelium and vessels with a structured wall (Fig. 4D). The initial development of the secondary ossification center in this series was only observed in the 5months-old proximal humerus (Fig. 4E). These results demonstrate the same chondrocyte maturation cycle as in zone 1 , but with a different ossification pattern.

The chondrocyte cytology during the course of the maturation cycle is characterized by cytoplasmic vacuole formation and size increase. Chondrocytes are smaller and round-shaped in fetal zone 3 , and their volume greatly increases in zones 2 and 1. Cells are stained by Alcian blue, no PAS positive deposits can be observed inside intracytoplasmic vacuoles. Nuclear pyknosis is commonly observed in zone 1 chondrocytes (Fig. 5).

\section{Light Microscopy Morphometry}

The TCLA in fetal Anlagen significantly increases from zones 4 and 3 of the epiphyses to zones 2 and 1 of the diaphyses $(P<0.001)$. The total intercellular matrix area significantly decreases from zone 4 to zone $1(P<0.001)$. The highest increments related to the chondrocyte lacunar area parameters between zones 2 and 1 correspond to the sector of the cartilage Anlage where mineral deposition in the matrix is occurring (Fig. 6A). In line with the above reported data, the ratio between the TCLA and total matrix area (TMC) decreases significantly from zone 1 to 4 (Fig. 6A). This corresponds to an increment of the CLN from zone 1 to 4 (Fig. 6B) and the 

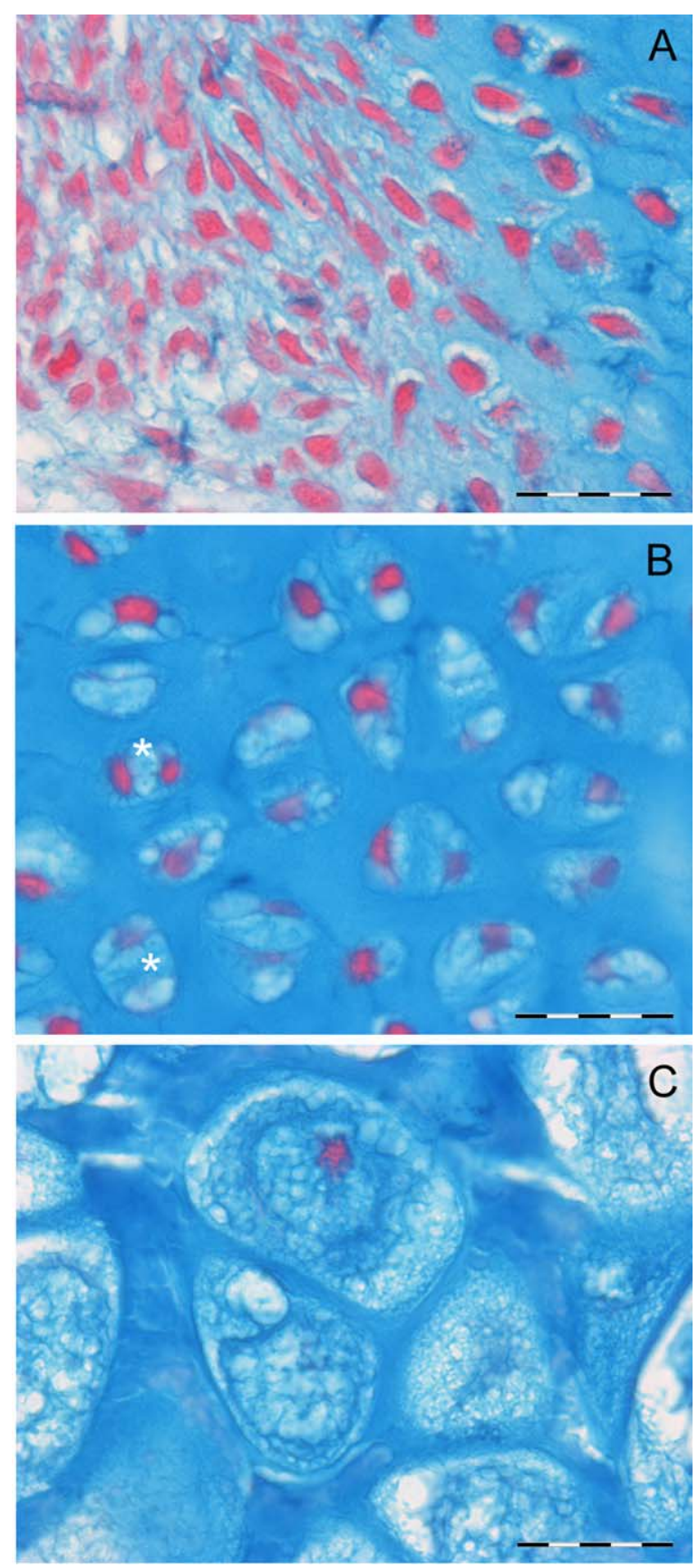

Fig. 5. Human fetal, 3rd metacarpus. (A) 20th week, (Alcian blue-neutral $\mathrm{red} / \mathrm{bar}=20 \mu \mathrm{m})$. Perichondrium and zone 4 chondrocytes characterized by a high rate of cell proliferation, small cell size and scanty intercellular matrix. (B) 20th week, 3rd metacarpal (Alcian blue-neutral $\mathrm{red} / \mathrm{bar}=20 \mu \mathrm{m}$ ). Chondrocyte maturation pattern in zone 3 , characterized by larger lacunar and cell size, small intracytoplasmic vescicles and increments of the intercellular matrix mass. The paired chondrocytes (asterisks) inside a unique lacuna are an evidence of cell intralacunar duplication which characterizes cartilage interstitial growth. (C) 20th week, 3rd metacarpal (Alcian blue-neutral red/bar $=20$ $\mu \mathrm{m})$. Swollen chondrocyte of zone 1 characterized by massive increment of the lacunar area. The cell inside is also enlarged due to dilated cisterns or cysts. Artifactual tears suggest a fluid content of the latter. Nuclei are picnotic. inverted relationship of the latter with the MSCLA (Fig. 6B).

Histological analysis of postnatal epiphyses (zone 5) with the corresponding fetal central epiphysis (zone 3) reveals a significantly lower TCLA and a higher TMA (both $P<0.001$ ) (Fig. 7A). Zone 5 exhibits a reduction in CLN with a concomitant increase in the MSCLA (Fig. 7A).

The hypertrophic cell layer in the postnatal growth plate (zone 6) exhibits TCLA values higher than those of fetal zone $2(P<0.01)$ and the TMA is significantly lower than that in zone $2(P<0.01$; Fig. 7B). The CLN is significantly lower in zone 6 than in zone $2(P<0.001)$ and the MSCLA is significantly higher $(P<0.001)$ between the same zones (Fig. 7B).

In summary, the morphometric analysis documented inversely related chondrocyte and matrix size parameters in fetal zone 4 and 1 . A similar trend was observable in postnatal metaepiphyses.

\section{Scanning Electron Microscopy}

Scanning electron microscopy observation of deparaffinized sections can be affected by technical artifacts because the cell membrane can partially detach from the lacunar border. Artefactual tears and shrinkage are present in most of zone 1 and zone 2 chondrocytes. However, a few cells that were still connected to the lacunar border were found. The large cytoplasmic vesicles appear empty after processing and their content remains unidentified, but should have originally held a well hydrated content (Fig. 8A). The intercellular matrix is formed by a reticular network of loose collagen fibrils in the epiphyses (zone 3; Fig. 8B). The network becomes more compact in zone 2 , and the fibrils appear tightly packed in zone 1, with patches of dense material deposed on the fibrils network (Fig. 8C). X-ray energy dispersive spectra reveal dense plaques with the typical calcium and phosphorus spectrum in accordance with zone 1 (Fig. 8D). No sign of calcium phosphate deposits was found in any field in zones 3 and 2 .

At higher magnification, the matrix interfibrillar empty space was observed to decrease gradually from zone 3 to 1 (Fig. 9A), which has been statistically analyzed (Fig. 9B).

The proximal humerus coplanar section of the postnatal 5-month-old meta-epiphysis, including both the secondary ossification center and the metaphyseal growth plate, directly demonstrates the hypertrophic chondrocyte structural pattern and the vascular network. The metaphyseal growth plate exhibits longitudinally organized columns with thin, parallel vascular spaces between the straight calcified cartilage septa (Fig. 4E). In the secondary ossification center, the same columns are arranged in a less orderly pattern, but 


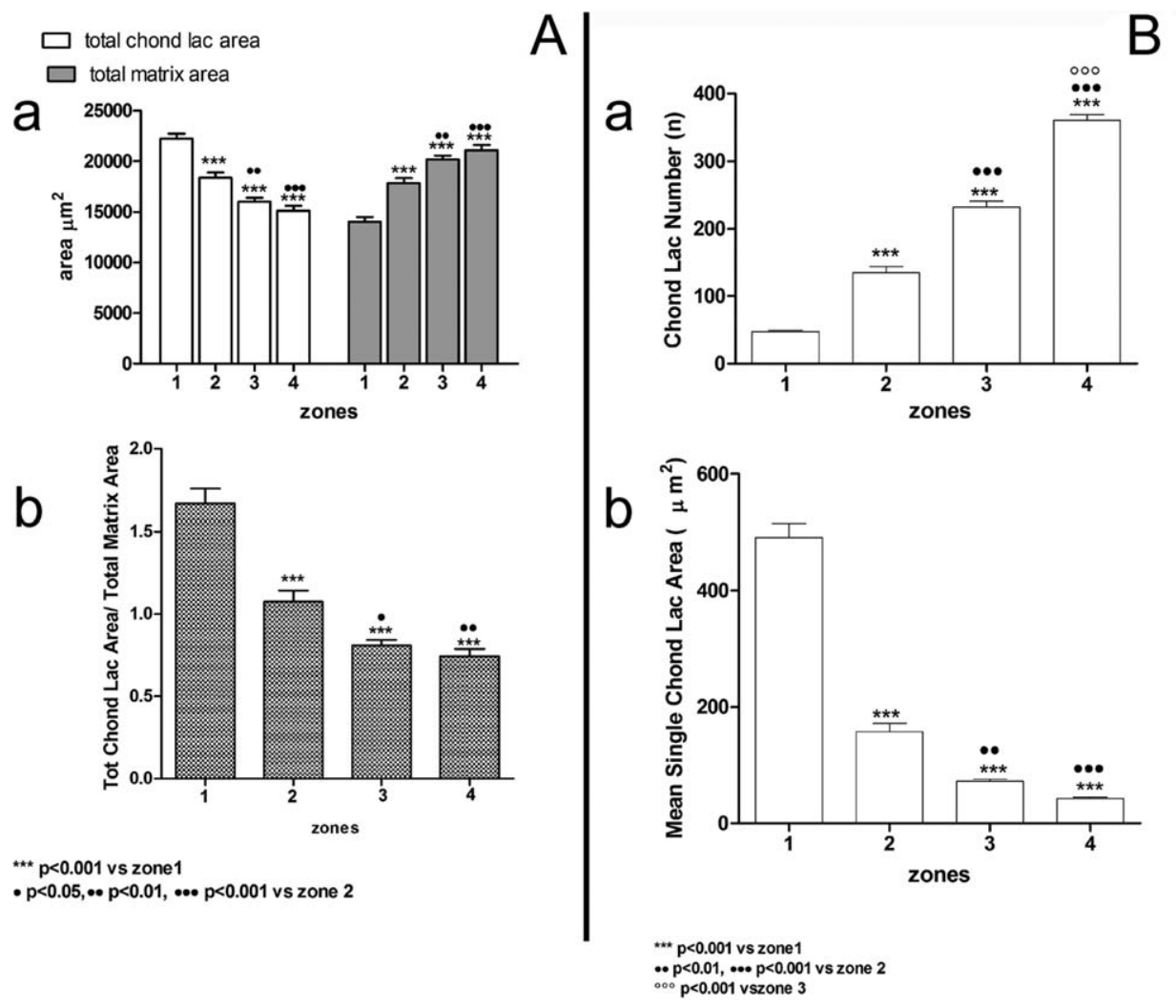

Fig. 6. (A) Histograms documenting the reciprocal variation of TCLA and TMA from zone 1 to 4 in fetal Anlagen (a). The ratio between the two paramenters significantly decreases from zone 1 to 4 (b). (B) Histograms documenting the increase in CLN from zone 1 to 4 (a) and the decrease in the MSCLA (b) in the same zones.

exhibit a recognisable pattern that converges centripetally, with vessels present in opposite direction emerging from a large, central vascular space (Fig. 4E). This vascular space is supplied by the vessels entering into the epiphysis through the cartilage canals network.

\section{DISCUSSION}

Enchondral bones of vertebrates develop from a cartilaginous Anlage in which the bone shape is already well defined before ossification begins (Uhthoff, 1990; Pazzaglia et al., 2011).

Earlier studies on the chondrocyte maturation cycle have been carried out in the metaphyseal growth plate cartilage (Buckwalter et al., 1986; Hunziker et al., 1987; Hunziker, 1994; Wilsman et al., 1996a, b; Ballock and O'Kneefe, 2003) and have focused on chondrocyte metabolic activity, shape/volume modulation and the relationship with the matrix mineralization. However, to the best of our knowledge, no research has yet been carried out in human fetal cartilage Anlagen to examine a morphological/morphometric basis for the above referenced biological processes and their relationship with the developmental patterns of primary and secondary ossification centers. These ossification centers develop from the diaphysis zone 1 and the epiphysis zone 3 of the cartilage Anlage with different dynamics and structural layouts compared to the metaphyseal growth plate. In particular, the chondrocytes of zone 1 (confined in a close environment that soon becomes surrounded by the periosteal sleeve) offer a characteristic model for the effects of fluid diffusion between the cells and the matrix.

The current study has some limitations:

1. Fetal age (16th-22nd week) and retrospective analysis of histological slides do not permit fetal length assessment. However, due to the developmental lag among Anlagen in the single fetuses, it was possible to pool the Anlagen according to Rivas and Shapiro (2002) histological staging. The rabbit gestation period is approximately 30 days compared to 9 months in humans, but an extrapolation of the staging is possible because the basic morphology and the sequence of the cartilage Anlage transformations do not differ between the species.

2. The orientation of cartilaginous Anlagen was obtained by selecting slides that included a complete, longitudinal section or at least half (midsector and epiphysis) of the Anlage. 


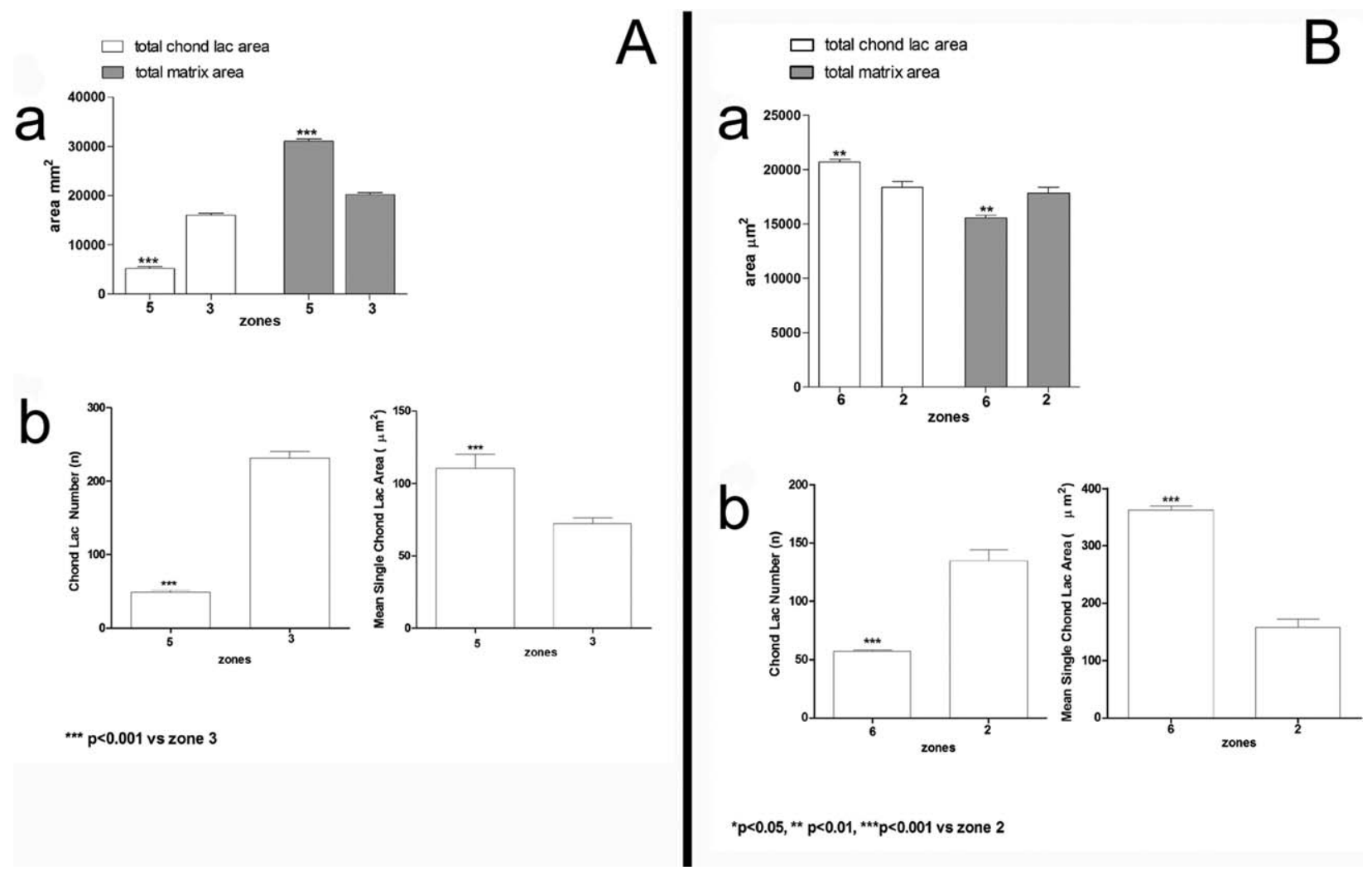

Fig. 7. (A) Histograms comparing the reciprocal variation of the TCLA and the TMA between Postnatal zone 5 and Fetal Anlage zone 3 (a). The CLN is significantly higher in Fetal zone 3 than in Postnatal zone 5, while the MSCLA is significantly higher in zone 5 than in zone 3 (b). (B) Histograms comparing the TCLA and the TMA between the postnatal metaphyseal growth plate cartilage (zone 6) and the cartilage Anlage zone 2 (a). The CLN and the MSCLA (b) demonstrate a significantly lower CLN and a higher MSCLA in postnatal zone 6 than in fetal zone 2.

3. One meta-epiphysis from each postnatal age $(2$, 3,3 , and 5 months) was longitudinally oriented before histological processing.

4. Artifacts occur due to processing of SEM deparaffinized sections, the same used for light microscopy. These were used mainly for morphology and morphometry of the matrix fibrils network. Despite the quality limitation of the SEM images, the method has the advantage of evaluating the same slides where light microscopy morphometry has been carried out.

\section{Chondrocyte Maturation Cycle and Matrix Transformation}

The inverse correlation of the chondrocyte lacunar area parameters with those of the interterritorial matrix suggests the uniqueness of cells and matrix transformations in the chondrocyte maturation cycle characterizing the first stage of endochondral ossification. The chondrocyte lacunar area variations in the passage from zone 3 to 2 could still be consistent with a concept of cell hypertrophy supported by a high matrix synthesis level and could eventually be associated to a certain degree with shrinkage of the fibrils network.
However, the changes in both the morphological and morphometric parameters from zone 2 to 1 clash with this hypothesis and are better explained by cell swelling. Earlier studies carried out in the metaphyseal growth plate cartilage (Buckwalter et al., 1986; Farnum et al., 2002) have considered this question; the first paper reported an increase in intracytoplasmic organelles of $126 \%$ from the proliferative zone to the hypertrophic zone against an increment of $779 \%$ for the total cell volume (cytoplasm + nucleus), and the second study reported chondrocyte lacunar area variation that was in agreement with the analysis of early postnatal zone 6 in the current study.

With regard to the cartilage permeability and the transport of solutes, the major fraction of cartilage water is reported to be free (Maroudas, 1975, 1976; Maroudas and Schneiderman, 1987; Torzilli, 1988); thus the molecular packing density and intrafibrillar water content are a function of the osmotic pressure difference between the extrafibrillar and intrafibrillar spaces (Maroudas et al., 1991). Considering the environmental condition of the Anlage zone 1 , the recovery of $\mathrm{H}_{2} \mathrm{O}$ into the swelling cell would correspond to the 

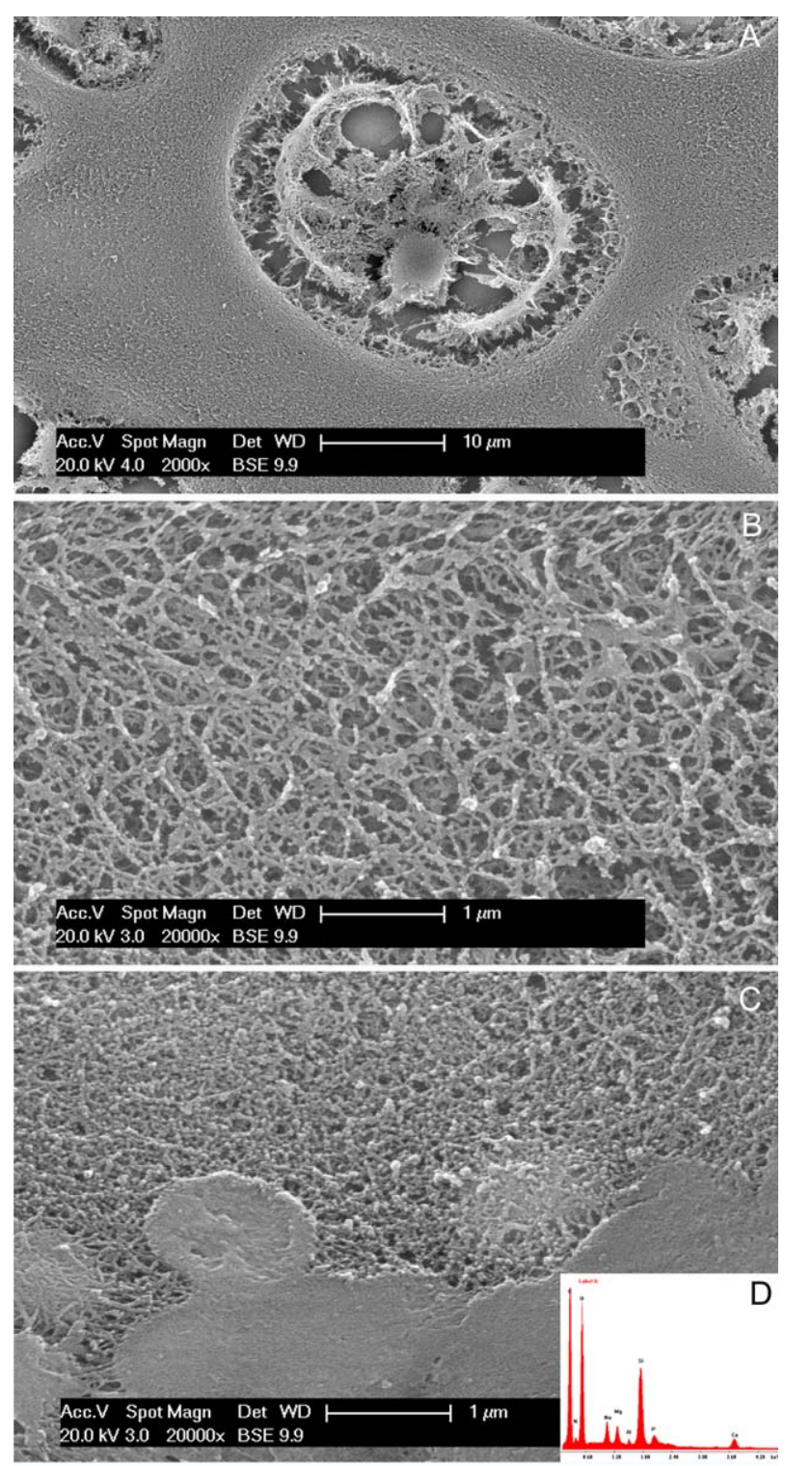

Fig. 8. Human fetal, 3rd metacarpus. 20th week, (SEM, backscattered). (A) The swollen chondrocytes of zone 1 had undergone to schrinkage and artifacts due to processing dehydration. In the figure, the cell has maintained (despite schrinkage) contact with the lacunar border along the whole perimeter. Cysts and cisterns are evident inside the cytoplasm. (B) Morphology of zone 3 cartilage intercellular matrix. Collagen fibrils dispersed in a 3-D reticulum. (C) Tightly packed collagen fibrils of zone 1 (compared to $\mathbf{B}$ ) and $\mathrm{CaP}$ nucleation in the bottom of the figure. Compacting was not an artifact of processing because images $\mathbf{B}$ and $\mathbf{C}$ were acquired from the same Anlage. (D) X-rays energy dispersive spectrum of the mineralized field in $\mathbf{C}$ documenting the $\mathrm{CaP}$ peaks.

concentration of $\mathrm{CaPO}_{4}$ in the matrix, up to a critical threshold of mineral precipitation. Different mechanisms of cartilage calcification induction have been suggested, such as matrix vescicles, or a family of calcium channel molecules denoted as annexins that are linked to type-II and type-X collagen (Mwale et al., 2002; Ballock and O'Kneefe,
2007; Bonucci 2007). However, the hypothesis of $\mathrm{H}_{2} \mathrm{O}$ recovery into the cell rather than the transport of calcium and phosphorous ions from the cell into the matrix aligns with the morphology and morphometry observed in this study. The calcification of the Anlage cartilage has a close relationship with the onset of endochondral ossification in the diaphyseal and epiphyseal ossification centers, but at a later age in the latter.

\section{Ossification Center Development}

The fetal epiphyses up to the 22nd week of age, even in the Anlagen with the most advanced histological developmental stage (upper limb proximal humeri) do not exhibit cartilage canals. In contrast, a well- developed network is evident in all postnatal epiphyses, indicating that canals have been progressively organized at least from the $23 \mathrm{rd}$ week onwards. To date, no morphological evidence indicates a causal relationship between canal system development and the secondary ossification center, only suggesting that the latter comes afterwards (Haraldsson, 1962; Gray and Gardner, 1969; Lufti, 1970; Wilsman and Van Sickle, 1970, 1972; Agrawal et al., 1986; Ganey et al., 1992, 1995; Burkus et al., 1993; Shapiro, 1998; Blumer et al., 2008). Indeed a reliable, systematic evaluation of the appearance of the secondary ossification centers can only be obtained by skeletal X-ray surveys (Vogt and Vickers, 1938, Noback, 1944; Christie, 1949; Greunlich and Pyle, 1959; Caffey, 1973). The observation that some canals only contain loose connective tissue without vascular differentiated structures and others contain vascular lacunae or even structured vessels supports the view that the system plays a primary role in providing nutrition to cartilage. Canals are advantageous in large-sized epiphyses for the diffusion of water and solutes into the inner core, but do not indicate that the canal presence alone could initiate the endochondral ossification process. The structured vessels can use the canal network to reach at the appropriate developmental period the epiphyseal ossification center.

\section{Structural Layout of Epiphyseal and Diaphyseal Ossification}

The epiphyseal ossification morphology demonstrates the already reported chondrocyte maturation cycle and the matrix calcification but with a centripetal progression and alignment of the hypertrophic cells. The vessels reach the ossification center through some of the pre-existing cartilage canals. They are branches of the external metaphyseal vascular network or even of the intramedullary vascular system (Brookes and Revell, 1998; Shapiro, 1998). There is a substantial difference between diaphyseal and epiphyseal 

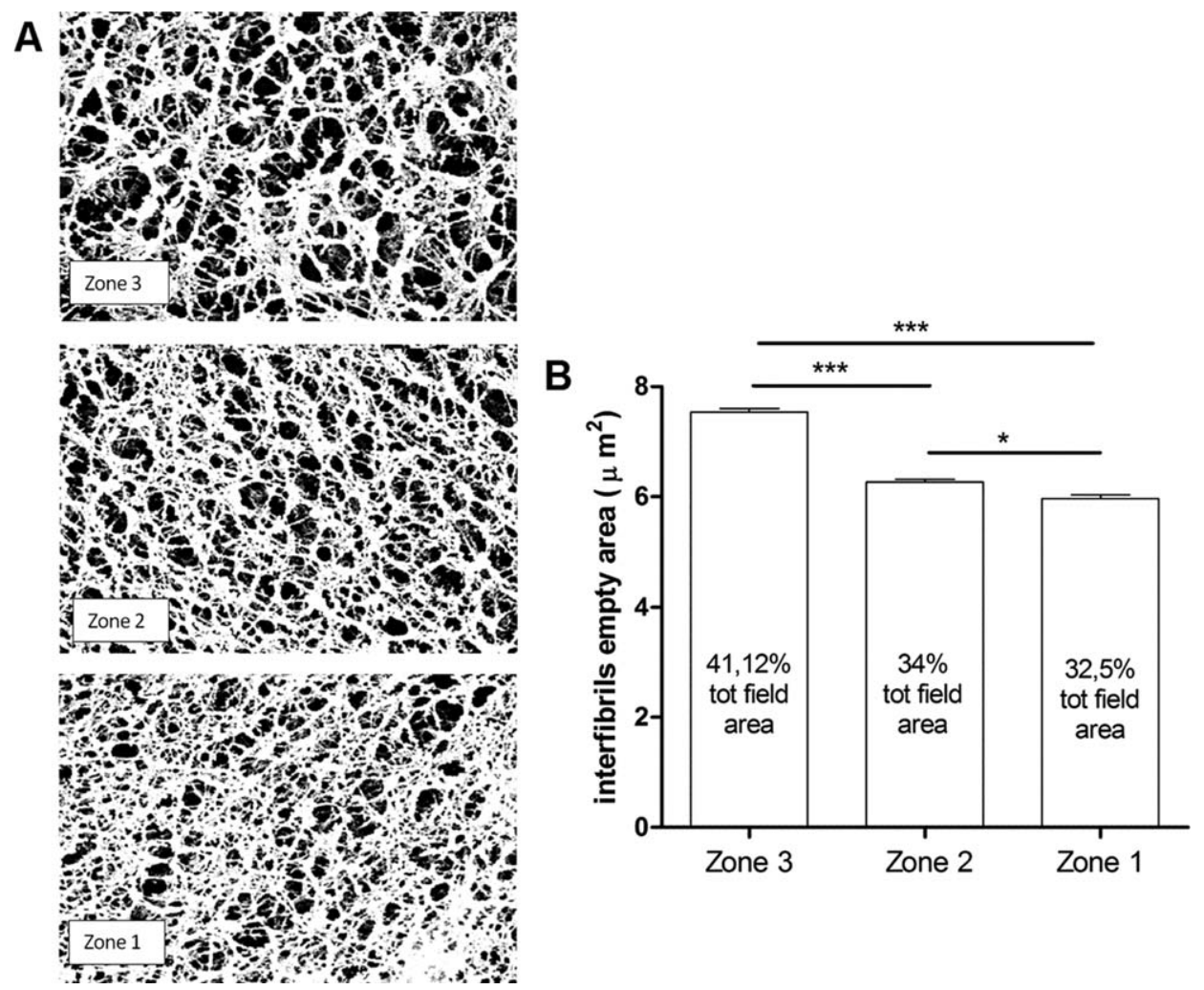

Fig. 9. Human fetal, 3rd metacarpus. 20th week (SEM backscattered, 25.000×). Interfibrils empty spaces in zone 3,2 , and 1 (A). The morphometric analysis of the interfibrils' empty area reveals a significant reduction from zone 3 to zone 2 and from zone 2 to 1 in the same Anlage.

ossification: the calcified cartilage of the former provides a single cylindrical scaffold for the periosteal apposition, which is almost completely resorbed by the intramedullary vascular network advancement. Epiphyseal and metaphyseal ossification are characterizeds by osteoblasts apposition on the thin calcified cartilage septa between the chondrocyte columns.

In conclusion, a common chondrocyte maturation cycle provides a stiff scaffold for osteoblast apposition, but ossification progresses with different patterns in the diaphysis, the epiphysis and the metaphysis. Its schedule and pattern appear to be set by the local morphology and the development of the vascular supply.

\section{ACKNOWLEGDMENTS}

SEM observation and EDAX analysis was carried out with an electron microscope of the "Department of Surgical and morphological Sciences" of the University of Insubria. The two anonymous reviewers and the editor are greatly appreciated for their valuable comments and suggestions in the revision of the manuscript.

\section{LITERATURE CITED}

Agrawal P, Kulkarni DDS, Atre PR. 1986. The participation of cartilage canals in the ossification of the human fetal calcaneum. J Anat 147:135-142.

Ballock RT, O'Keefe RJ. 2003. The biology of the growth plate. J Bone Joint Surg Am 85A:715-726.

Bland JM, Altman DG. 2010. Statistical methods for assessing agreement between two methods of clinical measurement. Int J Nurs Stud 47:931-6.

Blumer MJ, Longato S, Fritsch H. 2008. Structure, formation and role of cartilage canals in the developing bone. Ann Anat 190:305-15.

Bonucci E. 2007. Biological Calcification. Berlin: Springer-Verlag. pp. 261-301.

Brookes M, Revell WJ. 1998. Blood Supply of Bone. London: Springer-Verlag.

Buckwalter JA, Mower D, Ungar R, Schaeffer J, Ginsberg B. 1986. Morphometric analysis of chondrocyte hypertrophy. J Bone Joint Surg Am 68:243-55.

Burkus JK, Ganey TM, Ogden JA. 1993. Development of the cartilage canals and the secondary center of ossification in the distal chondroepiphysis of the prenatal human femur. Yale J Biol Med 66:193-202.

Caffey J. 1973. Pediatric X-Ray Diagnosis. London: Lloyd-Luke (medical books) LTD.

Christie A. 1949. Prevalence and distribution of ossification centers in the newborn infant. Am J Dis Child 77:355-61.

Farnum CE, Wilsman NJ. 1993. Determinations of proliferative characteristics of growth plate chondrocytes by labeling with bromodeoxyuridine. Calc Tissue Int 52:110-9.

Farnum CE, Lee R, O'Hara K, Urban JP. 2002. Volume increase in growth plate chondrocytes during hypertrophy: The contribution of organic osmolytes. Bone 30:574-81. 
Ganey TM, Love SM, Ogden JA. 1992. Development of vascularization in the chondroepiphysis of the rabbit. J Orthop Res 10:496-510.

Ganey TM, Ogden JA, Sasse J, Neame PJ, Hilbelink DR. 1995. Basement membrane composition of cartilage canals during development and ossification of the epiphysis. Anat Rec 241:425-37.

Gray DJ, Gardner E. 1969. The prenatal development of the human humerus. Am J Anatomy 124:431-45.

Greunlich WW, Pyle SL. 1959. Radiographic Atlas of Skeletal Development of the Hand and Wrist, 2nd ed. Stanford, CA: Stanford University Press.

Haraldsson S. 1962. The vascular pattern of a growing and fullgrown human epiphysis. Acta Anat (Basel) 48:156-67.

Hunziker EB. 1994. Mechanism of longitudinal bone growth and its regulation by growth plate chondrocytes. Microsc Res Tech 28:506-519.

Hunziker EB, Schenk RK, Cruz-Orive LM. 1987. Quantitation of chondrocyte performance in growth-plate cartilage during longitudinal bone growth. J Bone Joint Surg Am 69:162-173.

Lufti AM. 1970. Mode of growth, fate and function of cartilage canals. J Anat 106:135-145.

Maroudas A. 1975. Fluid transport in cartilage. Ann Rheum Dis 34:77-81.

Maroudas A. 1976. Transport of solutes through cartilage: Permeability to large molecules. J Anat 122:335-347.

Maroudas A, Schneiderman R. 1987. "Free" and "exchangeable" or "trapped" and "non-exchangeable" water in cartilage. J Orthop Res 5:133-8.

Maroudas A, Wachtel E, Grushko G, Katz EP, Weinberg P. 1991. The effect of osmotic and mechanical pressure on water partitioning in articular cartilage. Biochim Biophys Acta 1073:285-94.

Mwale F, Tchetina E, Wu CW, Poole AR. 2002. The assembly and remodeling of the extracellular matrix in the growth plate in relationship to mineral deposition and cellular hypertrophy: An in situ study of collagens II and IX and proteoglycan. J Bone Miner Res 17:275-283.

Noback CR. 1944. The developmental anatomy of the human osseus skeleton during the embryonic, fetal and circumnatal period. Anat Rec 88:91-125.

O'Rahilly R, Gardner E. 1972. The initial appearance of ossification in staged human embryos. Am J Anat 134:291-301.
Pazzaglia UE, Dell'Orbo C, Wilkinson MJ. 1987. The foreign body reaction in total hip arthroplasties. A correlated lightmicroscopy, SEM and TEM study. Arch Orthop Trauma Surg 106:209-219.

Pazzaglia UE, Beluffi G, Benetti A, Bondioni MP, Zarattini G. 2011. A review of the actual processes governing growth and development of long bones. Fetal Pediatr Pathol 30: 199-208.

Rasband, WS. 1997-2016. ImageJ, Bethesda, MD: U. S. National Institutes of Health. Available at: http://imagej.nih. gov/ij/

Rivas R, Shapiro F. 2002. Structural stages in the development of the long bones and epiphyses. J Bone Joint Surg Am 84A: 85-100.

Schneider CA, Rasband WS, Eliceiri KW. 2012. NIH Image to ImageJ: 25 years of image analysis. Nat Methods 9: 671-675.

Shapiro F. 1998. Epiphyseal and physeal cartilage vascularization: A light microscopic and tritiated thymidine autoradiographic study of cartilage canals in newborn and young postnatal rabbit bone. Anat Rec 252:140-148.

Streeter GI. 1951. Developmental Horizons in Human Embryos. Washington: Carnegie Institution of Washington.

Torzilli PA. 1988. Water content and equilibrium water partition in immature cartilage. J Orthop Res 6:766-769.

Uhthoff HK. 1990. The Embryology of the Human Locomotor System. Berlin: Springer-Verlag.

Vogt EC, Vickers VS. 1938. Osseous growth and development. Radiology 91:441-444.

Wilsman NJ, Van Sickle DC. 1970. The relationship of cartilage canals to the initial osteogenesis of secondary centers of ossification. Anat Rec 168:381-392.

Wilsman NJ, Van Sickle DC. 1972. Cartilage canals, their morphology and distribution. Anat Rec 173:79-93.

Wilsman NJ, Farnum CE, Green EM, Lieferman EM, Clayton MK. 1966a. Cell cycle analysis of proliferative zone chondrocytes in growth plates elongating at different rates. J Orthop Res 14:562-572.

Wilsman NJ, Farnum CE, Lieferman EM, Fry M, Barreto C. 1966b. Differential growth by growth plates as a function of multiple parameters of chondrocytes. J Orthop Res 14:927936. 